\title{
Matrix Model and Ginsparg-Wilson Relation
}

\author{
Keiichi Nagao ${ }^{\text {a* }}$ \\ ${ }^{a}$ The Institute of Particle and Nuclear Studies, \\ High Energy Accelerator Research Organization (KEK), Tsukuba 305-0801, Japan
}

We discuss that the Ginsparg-Wilson relation, which has the key role in the recent development of constructing lattice chiral gauge theory, can play an important role to define chiral structures in finite matrix models and noncommutative geometries.

\section{Introduction}

Various matrix models have been proposed toward the nonperturbative formulations of the superstring theory. IKKT matrix model[1] is one of the proposals, and its several properties have been investigated [2]. In the matrix model spacetime is described in matrices, so noncommutative (NC) geometry [3] naturally appears in itself. The realization of chiral fermion is one of the interesting issues from the standpoint of the unification of space-time and matter since the chiral structure of fermions may play important roles in quantization of space-time.

One way to realize the 4 dimensional chiral fermions in the matrix model is the Kaluza-Klein compactification with the non-trivial index. The construction of the non-trivial index in finite $\mathrm{NC}$ geometry or matrix model is thus an important subject. Topologically nontrivial configurations in finite NC geometries have been constructed based on algebraic K-theory and projective modules in much literature, but it would be better if we could find other prescriptions which are more natural in physical point of view. One of the possibilities is the utilization of the admissibility condition [4] and the Ginsparg-Wilson relation [5] in lattice gauge theory (LGT).

In LGT there occurred much development recently in the construction of chiral gauge theory. The first important observation was that, in the presence of the mass defect which is introduced

\footnotetext{
* This talk is mainly based on the work with H.Aoki (Saga Univ.) and S.Iso (KEK).
}

as a scalar background in higher $4+x$ dimensions, a chiral fermion appears at the defect. So far a domain wall fermion $(x=1)[\underline{6}$ and a vortex fermion $(x=2)$ [7] are constructed on the lattice. From the former model a practical solution to the GW relation [5], the overlap Dirac operator, is obtained [8. The index theorem [910] and the modified chiral symmetry 1011] are also realized, and eventually, an anomaly free abelian chiral gauge theory is constructed on the lattice 12].

On the other hand in Connes' NC geometry the formulation is evolved from the spectral triple $(\mathcal{A}, \mathcal{H}, \mathcal{D})$, where a chirality operator and a Dirac operator which anti-commute are introduced $[3]$. In ref. [13], we proposed to generalize the algebraic relation to GW relation [5] so that we can define chiral structures in a finite noncommutative geometry. We will discuss these topics briefly in the following.

\section{GW fermion on finite NC geometry}

First we overview the relevant works shortly.

The first literature related to this context is found in ref. [14], where overlap formula is applied to the unitary IIB matrix model. At this time, however, the real importance of the GW relation and the relation between them were not known yet. Next in ref. [15] GW relation on fuzzy 2 -sphere was discussed for free fermions in connection to the fermion doubling problems. On $\mathrm{NC}$ torus the lattice structure is visible [16], so the usual overlap Dirac operator $[8$ can be put naively on NC torus as it is. The authors in ref. 17] dis- 
cussed the chiral gauge theory on $\mathrm{NC}$ torus using the overlap Dirac operator. In the paper 18 the gauge field was introduced to the Dirac operator in ref. 15, but the modified chiral symmetry was not maintained since the gauge field was introduced linearly in the Dirac operator. Dirac operator which has the exact chiral symmetry and no doubling species for general gauge fields has not been realized until the appearance of the paper [13.

The prescription proposed in ref. 13 is as follows. First we introduce two hermitian chirality operators, $\Gamma$ and $\hat{\Gamma}=\frac{H}{\sqrt{H^{2}}}$, which satisfy $\Gamma^{2}=\hat{\Gamma}^{2}=1 . \quad H$ includes the differential operator and the gauge field. The Dirac operator $D_{G W}$ is defined by $1-\Gamma \hat{\Gamma}=f(a, \Gamma) D_{G W}$, where $a$ is a small parameter. $H$ and the function $f$ must be defined so that the $D_{G W}$ has no doublers and the correct behavior in the commutative limit $(a \rightarrow 0) . \quad D_{G W}$ satisfies GW relation [5], $\Gamma D_{G W}+D_{G W} \hat{\Gamma}=0$, and we have the index theorem: index $D_{G W} \equiv\left(n_{+}-n_{-}\right)=$ $\frac{1}{2} \mathcal{T} r(\Gamma+\hat{\Gamma})$. Under the modified chiral transformation $\delta \Psi=i \lambda \hat{\Gamma} \Psi, \delta \bar{\Psi}=i \bar{\Psi} \lambda \Gamma$, the fermionic action $S_{F}=\operatorname{tr}\left(\bar{\Psi} D_{G W} \Psi\right)$ is invariant, while the Jacobian produces $q(\lambda)=\frac{1}{2} \mathcal{T} r(\lambda \hat{\Gamma}+\lambda \Gamma)$, which is expected to provide the topological charge density and the index for $\lambda=1$.

\subsection{Example on fuzzy 2-sphere}

We give the concrete example of $H$ and $f$ for fuzzy 2 -sphere case setting the NC coordinates as $x_{i}=\alpha L_{i}$, where $L_{i}$ 's are $2 L+1$ dimensional irreducible representation matrices of $S U(2)$ algebra. The radius of the sphere is then given by $\rho=\alpha \sqrt{L(L+1)}$. One example set of $H$ and $f$ are $H=\Gamma^{R}+a D_{w}$ and $f=-a \Gamma^{R}$, where $a=1 /(L+1 / 2)$ and $R$ means that the operator acts from the right on the matrices. $D_{w}$ and $\Gamma^{R}$ are given by $D_{w}=\sigma_{i}\left(\mathcal{L}_{i}+\rho a_{i}\right)+1$ and $\Gamma^{R}=\frac{1}{2 L+1}\left(2 \sigma_{i} L_{i}^{R}-1\right)$, where $i$ runs from 1 to 3. $a_{i}$ 's are gauge fields and $\mathcal{L}_{i}$ 's are Killing vectors satisfying $\mathcal{L}_{i} M=\left[L_{i}, M\right]$ for any hermitian matrix $M$. We denote here the Dirac operator constructed from these $H$ and $f$ as $D_{A I N}$.

We can show that the Chern-character is correctly produced from the Jacobian in the com- mutative limit; $q(\lambda)=2 \rho \int \frac{d \Omega}{4 \pi} \lambda \epsilon_{i j k} x_{i} \partial_{j} a_{k}^{\prime}$ where $a_{i}^{\prime}$ is a tangential component of $a_{i}: \quad a_{i}^{\prime}=$ $\epsilon_{i j k} x_{j} a_{k} / \rho[13]$.

The properties of $D_{A I N}$ and other types $D_{W W}$ and $D_{G K P}$ are summarized in Table 1. $D_{W W}$, $D_{G K P}$ and their properties related to doublers are seen in refs. [19], [20] and 2115], respectively. Here we note that $D_{w}$ is equal to $D_{G K P}$ and that $D_{W W}$ has no chiral anomaly. The source of the chiral anomaly in $D_{G K P}$ is the breaking in a cut-off scale of the action under the chiral transformation 22, while that in $D_{A I N}$ is the Jacobian 13. These facts suggest that some kind of Nielsen-Ninomiya's theorem exists in matrix model and NC geometry.

\section{Non-trivial index on fuzzy 2-sphere}

Based on the construction in ref. 13, a concrete example of the non-trivial index is constructed with the projective module method in ref. 23]. Using the same method we can construct the TP monopole on fuzzy 2-sphere 24]. However, the index operator is modified and the original interpretation is lost. This is an open problem.

\subsection{Example on $\mathrm{NC}$ torus}

GW fermion on NC torus is defined with the overlap Dirac operator 8 in LGT as it is [17. In addition to the usual chirality operator $\gamma_{d+1}$, the other one $\hat{\gamma}=\frac{H}{\sqrt{H^{2}}}$ is introduced. Here $H=\gamma_{d+1}\left(m_{0}-a D_{\mathrm{w}}\right)$ with the Wilson-Dirac operator, $D_{\mathrm{w}}=\frac{1}{2}\left[\gamma_{\mu}\left(\nabla_{\mu}^{*}+\nabla_{\mu}\right)-\operatorname{ar} \nabla_{\mu}^{*} \nabla_{\mu}\right]$, where $m_{0}$ and $r$ are free parameters. Using the above chirality operators, the GW Dirac operator is expressed as $D_{G W}=\frac{1}{a}\left[1-\gamma_{d+1} \hat{\gamma}\right]$, which is free of species doubling if $0<\frac{m_{0}}{r}<2[8]$.

The Jacobian is shown to produce the correct chiral anomaly in arbitrary dimensions $(d=2 n)$ using the result in ref. 25]. The final expression is written as $q(\lambda)=\frac{(-1)^{n}}{(4 \pi)^{n} n !} \epsilon_{\nu_{n} \mu_{n} \cdots \nu_{1} \mu_{1}} \int d^{d} x \lambda(x) \star$ $F_{\nu_{n} \mu_{n}}(x) \star \cdots \star F_{\nu_{1} \mu_{1}}(x)[26]$.

\section{Non-trivial index on NC torus}

In LGT the non-trivial topological structure in gauge field space is realized by the admissibility condition [4, which requires that the fluctuation of the gauge field strength is sufficiently small. 
Table 1

The properties of three types of Dirac operators on fuzzy 2-sphere

\begin{tabular}{|c|l|c|c|c|}
\hline Dirac operator & \multicolumn{2}{|c|}{ chiral symmetry } & no doublers & counterpart in LGT \\
\hline$D_{W W}$ & $D_{W W} \Gamma+\Gamma D_{W W}=0$ & $\bigcirc$ & $\times$ & naive fermion \\
\hline$D_{G K P}$ & $D_{G K P} \Gamma+\Gamma D_{G K P}=\mathcal{O}(1 / L)$ & $\times$ & $\bigcirc$ & Wilson fermion \\
\hline$D_{A I N}$ & $D_{A I N} \hat{\Gamma}+\Gamma D_{A I N}=0$ & $\bigcirc$ & $\bigcirc$ & GW fermion \\
\hline
\end{tabular}

On $\mathrm{NC}$ torus GW fermion includes the gauge field in the compact form, so it is expected that the non-trivial topology may be realized with the condition not appealing to projective modules. The index can be studied by the spectral flow method. The gauge field configurations which satisfy the admissibility condition and produce non-trivial indices are indeed found numerically on NC torus 27. This result shows that the nontrivial topological structure is naturally realized in the gauge field space on $\mathrm{NC}$ torus.

\section{Discussion}

$\mathrm{GW}$ relation has important roles in matrix model as well as in LGT. It is desirable to construct many examples of GW fermions on various higher-dimensional NC geometries. We hope that not only the topology of gauge field space but also that of space-time can be classified with the index operator or its generalization.

\section{REFERENCES}

1. N. Ishibashi, H. Kawai, Y. Kitazawa and A. Tsuchiya, Nucl. Phys. B 498, 467 (1997).

2. For a review, see H. Aoki, S. Iso, H. Kawai, Y. Kitazawa, A. Tsuchiya and T. Tada, Prog. Theor. Phys. Suppl. 134, 47 (1999).

3. A. Connes, Noncommutative geometry, Academic Press, 1990.

4. M. Luscher, Commun. Math. Phys. 85, 39 (1982).

5. P. H. Ginsparg and K. G. Wilson, Phys. Rev. D 25, 2649 (1982).

6. D. B. Kaplan, Phys. Lett. B 288, 342 (1992); Y. Shamir, Nucl. Phys. B 406, 90 (1993).

7. K. Nagao, Nucl. Phys. B 636, 264 (2002); H. Neuberger, hep-lat/0303009
8. H. Neuberger, Phys. Lett. B 417, 141 (1998); Phys. Rev. D 57, 5417 (1998); Phys. Lett. B 427, 353 (1998).

9. P. Hasenfratz, V. Laliena and F. Niedermayer, Phys. Lett. B 427, 125 (1998).

10. M. Lüscher, Phys. Lett. B 428, 342 (1998).

11. F. Niedermayer, Nucl. Phys. Proc. Suppl. 73, 105 (1999).

12. M. Lüscher, Nucl. Phys. B 549, 295 (1999).

13. H. Aoki, S. Iso and K. Nagao, Phys. Rev. D 67, 085005 (2003).

14. N. Kitsunezaki and J. Nishimura, Nucl. Phys. B 526, 351 (1998).

15. A. P. Balachandran, T. R. Govindarajan and B. Ydri, hep-th/0006216

16. J. Ambjorn, Y. M. Makeenko, J. Nishimura, R. J. Szabo, JHEP 0005, 023 (2000).

17. J. Nishimura and M. A. Vazquez-Mozo, JHEP 0108, 033 (2001).

18. G. Immirzi and B. Ydri, hep-th/0203121

19. U. Carow-Watamura and S. Watamura, Commun. Math. Phys. 183, 365 (1997).

20. H. Grosse and P. Presnajder, Lett. Math. Phys. 33, 171 (1995); H. Grosse, C. Klimcik and P. Presnajder, Commun. Math. Phys. 178, 507 (1996); S. Iso, Y. Kimura, K. Tanaka and K. Wakatsuki, Nucl. Phys. B 604, 121 (2001).

21. A. P. Balachandran, T. R. Govindarajan and B. Ydri, Mod. Phys. Lett. A 15, 1279 (2000).

22. H. Aoki, S. Iso and K. Nagao, Phys. Rev. D 67, 065018 (2003).

23. A. P. Balachandran and G. Immirzi, hep-th/0301242

24. H. Aoki, S. Iso and K. Nagao, to appear.

25. T. Fujiwara, K. Nagao and H. Suzuki, JHEP 0209, 025 (2002).

26. S. Iso and K. Nagao, Prog. Theor. Phys. 109, 
1017 (2003).

27. K. Nagao, work in progress. 